\title{
Design of Impedance Measurement Module for an EEG and EIT Integrated System
}

\author{
Harsh Sohal $^{1}$, Bishal Karki ${ }^{2}$, Anjali Sharma ${ }^{3}$ and Youssoufa Mohamadou ${ }^{4}$ \\ ${ }^{1}$ Assistant Professor Dept. of ECE, Jaypee University of Information Technology \\ Waknaghat, India \\ ${ }^{2}$ University of LEEDS, London, United Kingdom \\ ${ }^{3}$ Assistant Professor, Dept. of ECE, AP Goyal Shimla University, India \\ ${ }^{4}$ Biomedical Engineering Department, Universite des Montagnes \\ harshsohal@gmail.com,bishal86@gmail.com,anjali.iitt@gmail.com, \\ usufcom@hotmail.com
}

\begin{abstract}
Electrical impedance tomography (EIT) has been performed with the electroencephalogram (EEG) reduce errors associated with the inverse problem due to unknown conductivity distribution in the brain. EIT is known to have the potential to produce images for detecting brain activities e. g. epileptic seizures, as a means complementary to EEG. Since EEG signal is generated from internal sources in the brain and EIT voltage signal is induced from externally injected current, the measurement system requires high temporal resolution to measure fast EIT signals and high resolution ADC for small EEG signals in order to measure EEG signals concurrent with EIT signals. We proposed the new design of EEG and EIT integrated system based on multiple channels of upgraded impedance measurement module (IMM3). IMM3 has adopted a direct digital synthesizer (DDS) to generate injection current. Using internal PLL, we could generate fast acquisition timing signal for EIT measurement. We used a 24 bit ADC with 2.5 MSPS measurement speed. Real and imaginary components of EIT signal could be calculated by the phase sensitive demodulation or FFT method. EEG data was decimated to improve the signal quality and to reduce the amount of data to be recorded. Other features were inherited from previous impedance measurement module (IMM2.5) including operation mode for external triggering and cascaded connection with EIT systems, the selfcalibration to maintain the performance, multiplexing, and phase sensitive demodulation. This document describes the design of the IMM3 for EEG and EIT integrated system in detail and presents the basic performance indices of tested module. It can be assembled to configure a multi-channel EEG and EIT integrated system.
\end{abstract}

Keywords: Electrical impedance tomography (EIT), electroencephalogram (EEG), impedance measurement module (IMM)

\section{Introduction}

Electrical impedance distribution of tissues can be observed using an image produced by Electrical impedance tomography (EIT). This can be achieved by placing electrodes on that part of body for which observation have to be taken. These electrodes are used for injecting currents and measuring voltages which can be further used for impedance computation. For EEG source imaging, impedance distribution of the brain should be known. The impedance measurements done at a time may not be same when measured at some other time. EIT has the potential to provide real-time in-vivo resistivity map of the brain, which may be used for real time source imaging in the EEG. Hybrid imaging systems have been proved to be

Received (February 9, 2018), Review Result (May 10, 2018), Accepted (May 26, 2018) 
advantageous for imaging human brain function [1]. EIT is used to measure tissue conductivity or changes in tissue conductivity. These imaging systems do not provide the imaging of cells which are in the acute ischemic state hence not enabling us to rescue them. This leads the need of monitoring of stroke patients using the modalities which are capable of imaging ischemia and hemorrhage simultaneously without interfering with each other's functionalities. The integrated measurement of EEG and EIT is considered to have the potential to accomplish this. EEG measures the difference of voltage on two locations on the scalp and hence is a bipolar measurement. The current is injected through a pair of electrodes and the potential difference measurement is done across another pair of electrodes in EIT [2].

In the conventional EEG source imaging, inverse problem is used to find out the position of source and magnitude changes in the brain. Conductivity values can be different for different kind of tissues and in EEG measurement position of EEG source is dependent prior knowledge of tissue resistivities. Resistivity distribution of the brain should be known in order to improve the accuracy for EEG source imaging. The resistivity measurements may vary with time. EIT has the potential to provide real-time in-vivo resistivity map in the brain, which may be used for real time source imaging in the EEG [3]. Simulation studies of the simple frequency difference method and weighted frequency difference method have proved that weighted frequency difference method is robust of the two [4].

\section{Literature Review}

For evaluation of the EIT systems resistor phantoms are used and tests are performed for performance evaluation and comparisons of various EIT systems [5]. Amplitude of the relative impedance change and local noise distribution are measured and compared. EIT is relatively a less expensive imaging technique, the subjects are not exposed to harmful radiations, and has a very high temporal resolution. This makes possible the rapid collection of data, and long term monitoring of the physiological changes of the living tissue. The tissue characterization can also be made using EIT [6]. Sheffield Mk3.5 EIT/EIS system measures the real and imaginary part of impedance on a frequency ranging from $2 \mathrm{kHz}$ to 1.6 MHz. It used eight electrodes with an adjoining drive/receive electrode data gain protocol. Eight identical DSP based data acquisition boards are there to generate the drive frequencies and obtain Fast Fourier Transform (FFT) of the acquired signal data [7]. An efficient low cost EIT system with the capability of 2-D imaging has also been reported [8]. Ultrasound refection signals are known to be good at locating sharp acoustic density charges at the object boundary, whereas EIT provides us of the information of the whole region. Combination of ultrasound information and EIT has been proved to provide us with a faster and more accurate EIT image reconstruction [9]. The development of a multifrequency EIT system, consisting of one current source and multiple voltmeters provides was explored to improve the SNR and reciprocity error. Initially the system was designed for acquiring brain signal for frequency range from $10 \mathrm{~Hz}-500 \mathrm{kHz}$. The performance of this EIT system is evaluated in terms of a CMR ratio, $\mathrm{SN}$ ratio, and errors [10]. The complex time difference image reconstruction algorithm is evaluated in relation to complex conductivity spectra [11].

3 dimensional image reconstruction using conductivity and permittivity measurements using mfEIT has also been explored [12]. A multi frequency time difference imaging algorithm based on the concept of homogenous complex conducted of three different frequencies is evaluated to provide reliable images of canine and human lungs [13]. Functions of fully parallel multi-frequency EIT system implemented using FPGAs are evaluated by performing phantom and animal imaging experiments. Fats multi-frequency imaging is demonstrated algorithm ECG gated EIT imaging [14]. The FPGA-based wireless LAN linked multi-channel EIT data acquisition system (DAS), fully programmable and reconfigurable from the PC, named as OXBACT-5 has also been presented. It has 16 
current injection channels and 64 voltmeters. [15]. Two 16-electrode electrical impedance tomography (EIT) current patterns has been compared in terms of their ability to reconstruct and quantify small amounts of bleeding inside a neonatal human head using both simulated and phantom data [16]. Simultaneous acquisition of EIT and EEG is proposed to have the potential to become a technique for imaging on telemetry units alongside EEG [17]. Two different conductivity imaging methods i.e., EIT and Magnetic Resonance Electrical Impedance Tomography (MREIT) have been reviewed by the authors in the background of neuro-imaging. A hybrid approach including EIT, EEG, MREIT and MRI has been introduced [18].

A system design to remove the difficulties such as time consumed in placing the electrodes, electrode contact impedance etc. is proposed, known as active electrode-based system. The electrode belt is used in this system which consists of 32 active electrodes [19]. A new EIT system called the KHU Mark2.5 with automatic self-calibration has been introduced with a new IMM module which can efficiently work in cooperation with other devices by a synchronization signal. This introduced the KHU Mark2.5 EIT system can be used for intra-channel and inter-channel calibrations of all voltmeters in multiple IMMs [20]. A hybrid device is introduced which is able to measure different tomographic signals based on the Magnitude Ratio and Phase Difference Detection method with measuring frequency ranging from $1 \mathrm{kHz}$ to $1 \mathrm{MHz}$ [21].

\section{Preliminary Experiments}

We have conducted some preliminary experiments for measuring EEG and EIT together. We used separate set of electrodes for EEG and EIT based on 10-20 system. Clinical EEG system was used for measuring EEG signals from the human subject shown in Figure 1. At the same time, we operated KHU Mark1 EIT system to acquire EIT data from different electrodes. The measured EEG signal included large EIT interference signal, so we could not determine EEG signal from the EIT and noise. Also, both systems were not synchronized. Filtering and other processing techniques were also not efficiently implemented in the measurement set up. Using different electrodes for EIT and EEG measurement, it's more difficult to analyze the results. This integrated EEG and EIT measurements have been done by separated EEG and EIT electrodes based on the 10-20 system. EEG signal is measured using BrainAmp MR (Brain Products, Germany). EIT signal is measured by KHU Mark1 EIT system which is a 16 channels system. This was an asynchronous recording and measurements are affected by the applied signal from an EIT measurement system. Figure 2(a) shows that how the electrodes of EEG + EIT integrated system can be placed on the human head based on EEG 10-20 electrode system. The date acquired by a signal channel upgraded IIM is shown in Figure 2(b) where amplitude varies with respect to frequency.

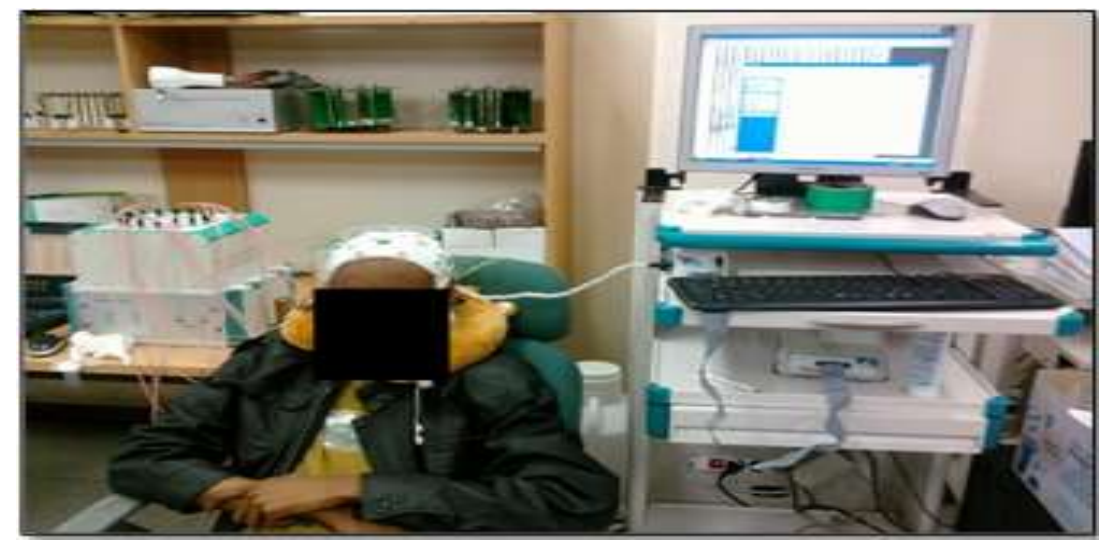

Figure 1. 16-channel KHU Mark1 EIT System 


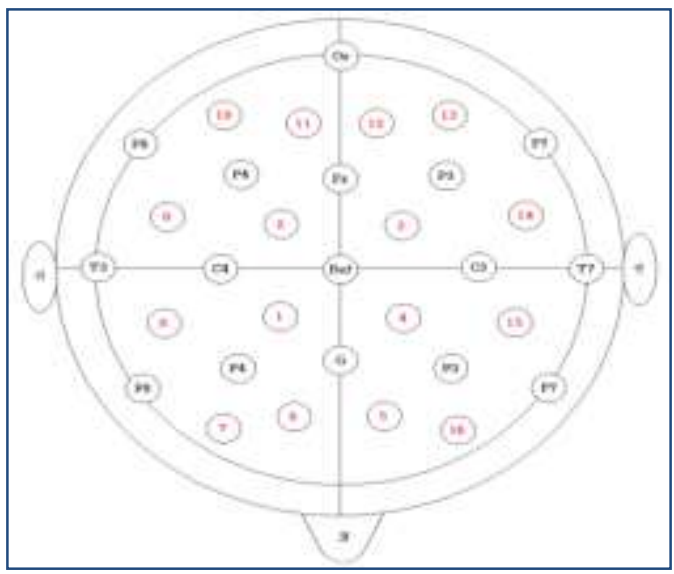

(a)

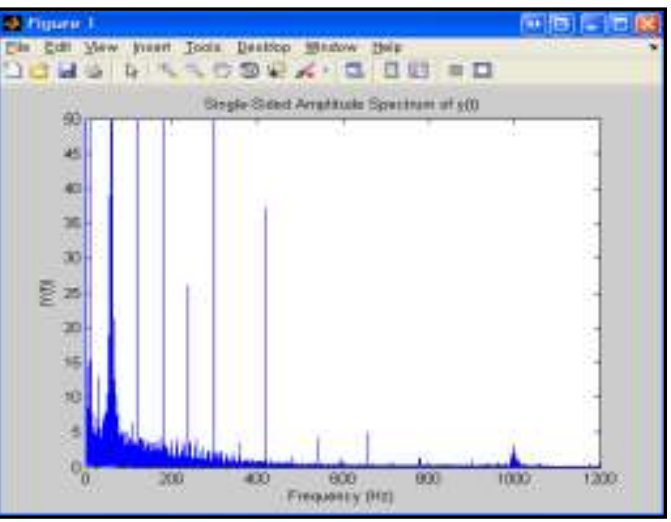

(b)

Figure 2. (a) EEG + EIT Electrodes (b) EEG + EIT Combined Signals

\section{EEG and EIT Integrated System}

We designed EEG and EIT integrated system to share same set of electrodes for measuring the EEG and EIT signal simultaneously as shown in Figure 3. EEG signal has $0.5-150 \mathrm{~Hz}$ frequency components, but EIT has the specific high frequency for applied signal. The magnitude of EEG is 10 or 100 times smaller than EIT signal. For simultaneous measurements, EEG is significantly interfered from EIT injecting signal and this artifact is also varied by the position of injection pairs. EIT data has additional effect from the EEG measurement circuits. To remove them, the easiest way is to measure EIT and EEG alternatively in time shared manner. But it is not optimized for detecting fast neural signal and impedance changes in the brain.

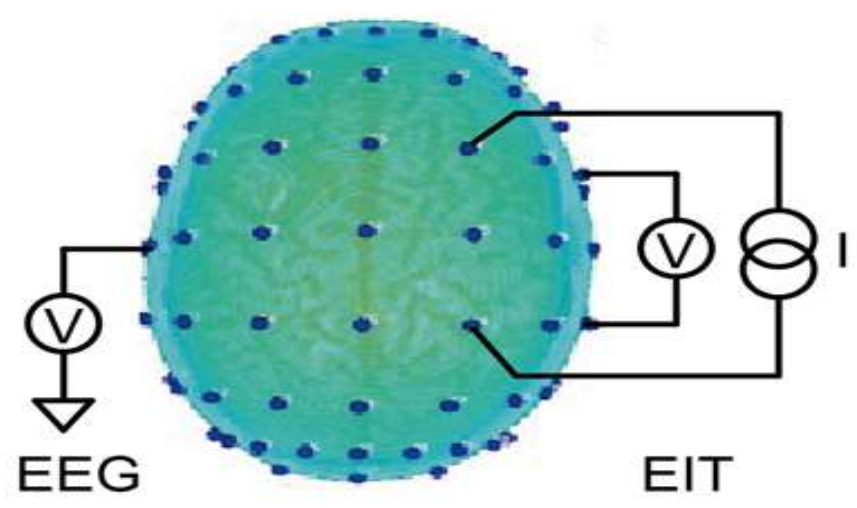

Figure 3. Integrated Measurement of EEG and EIT
EEG signal:
$(0.5-150) \mathrm{Hz}, \quad(5-300) \mu \mathrm{V}$

EIT signal:

$$
(1-100) \mathrm{kHz}, \quad \text { tens of } \mathrm{mV}(10-100 \text { times bigger than EEG signal })
$$

\section{Methodology}

\subsection{Objectives}

Integrated system design has been done to achieve following objectives: First objective is to model the brain activity by making a current dipole as an EEG source. As integrated EEG and EIT system can provide more information about the human tissues by performing complementary imaging, so there was a need of summed EEG and EIT signal. Second objective to make the system able to demonstrate that the EEG and EIT signals can be 
acquired simultaneously without corrupting each other and can be separated using available digital signal processing techniques. Third objective is processing of combined signal for accurate measurement. This combined signal may be further processed independently/parallel or iteratively (time multiplexed manner) to improve the measurements of one another. Particularly the EIT measurement has the potential to improve the EEG signal measurements by providing the real time conductivity measurements which may further be used in real time to model and observe the EEG sources inside the brain.

\subsection{System Architecture}

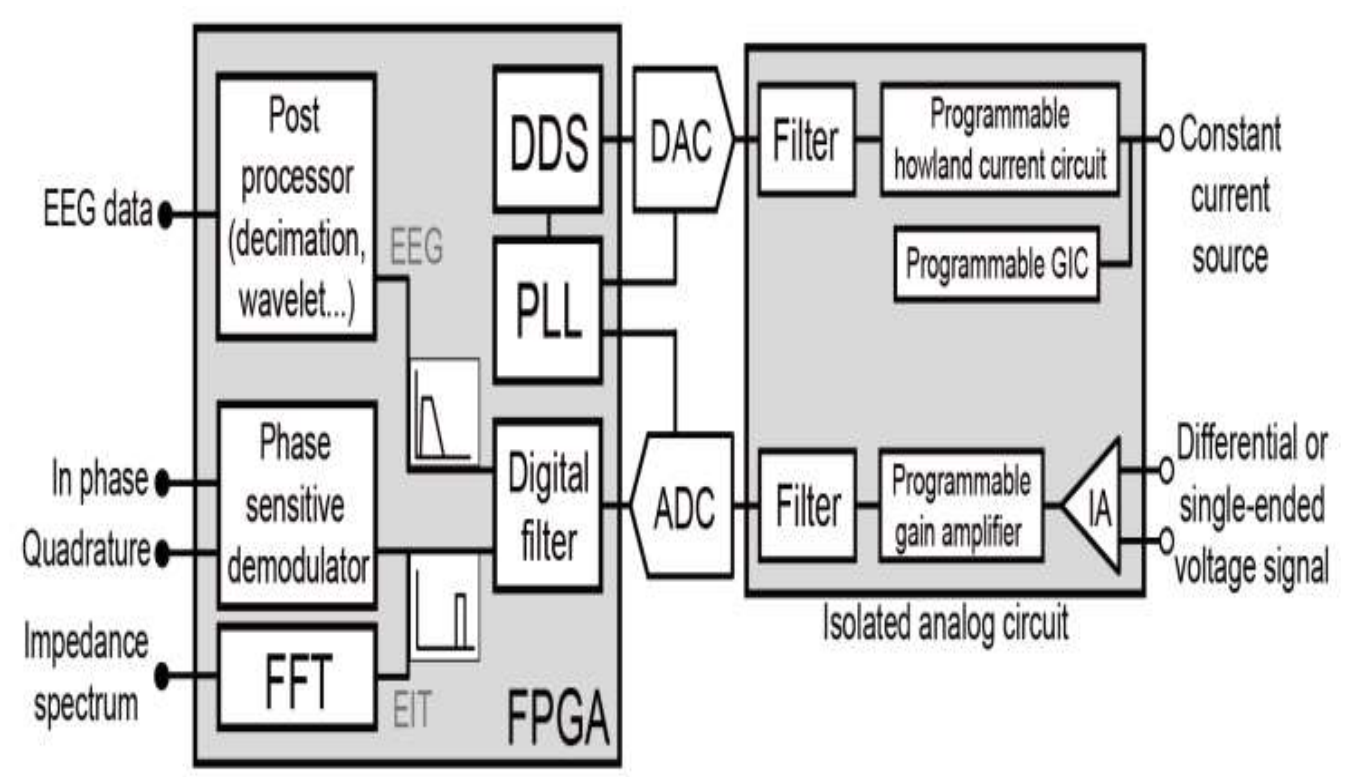

Figure 4. System Architecture for Impedance Measurement Module

The block diagram structure of the new IMM is shown in Figure 4. Isolated analog circuit is optimized for measuring EEG and EIT signal. This is equipped with high input impedance, small noise and wideband pass filter to pass both the target signal ranges. Use of DDS, provides us with extra flexibility to choose the operating frequency for EIT and also acquisition speed. Timing control logic based on the PLL can provide high speed digital processing such as filtering and special functions as wavelets and others. The ADC acquires data with a high speed and high resolution. This large amount of data from high speed data acquisition is decimated to reduce the data size and improve data quality for EEG. Other post processing parts are still required to be added and tested to improve the results.

\subsection{Impedance Measurement Module (IMM)}

Figure 5 shows the printed circuit board of an IMM with its various functional blocks. This is an implementation of new IMM, which is assembled together on the printed circuit board for EEG and EIT integrated measurement. DAC can compensate DC output with high resolution of 16 bits whereas ADC used has 24 bit max resolution with max sampling frequency up to 2.5MHz. Higher EIT operating frequency is used to separate EEG and EIT signal in the frequency domain. Stable power and high input impedance of amplifier affect the results positively. Main functional block of the IMM module are

- DAC: AD9783 (16bit, 2 channel output s+ 2 auxiliary outputs, maximum $600 \mathrm{MHz}$ sampling rate) 
- $\quad$ ADC: AD7760 (24bit, $2.5 \mathrm{MHz}$ sampling rate)

- FPGA: EP3C10F256C8N

- Op-Amp: OPA4140 (High input impedance - $10^{13} \Omega$, low bias current) and a stable customized power supply

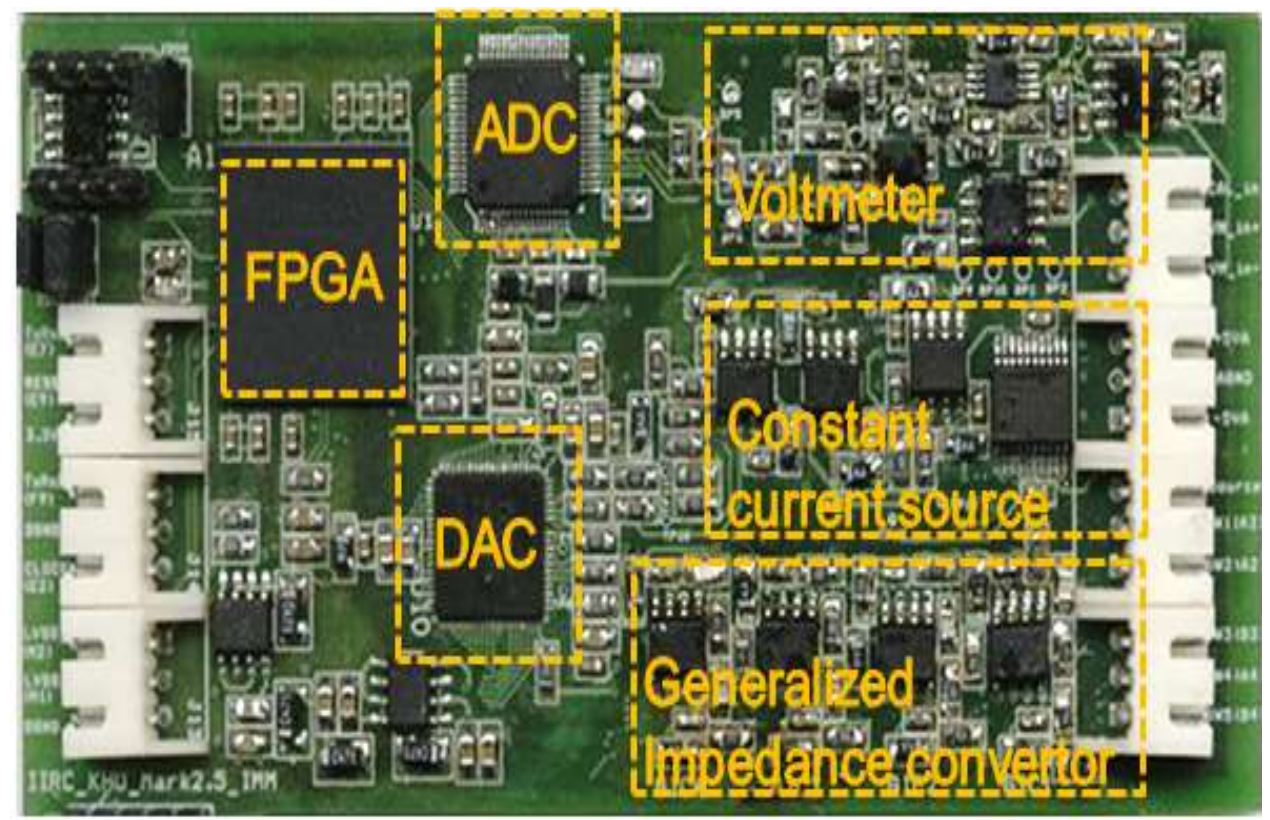

Figure 5. System Architecture for the IMM

\subsection{Apparatus Required to Achieve the Objectives}

Required apparatus to achieve the integrated EEG and EIT are Function generator, 16 channel EIT, cylindrical phantom [22-23], saline solution of fixed conductivity $(0.3 \mathrm{~S} / \mathrm{m})$, stand, modeled EEG signal source, injection electrodes, agar $(0.2[\mathrm{~S} / \mathrm{m}])$, EIT frequency generator of $1.125 \mathrm{KHz}$ (EEG+EIT IMM board), EEG+EIT IMM board experimental set up with digital backplane, main controller board, analog board for testing a single IMM, power supplies: $5 \mathrm{~V}$ digital to the main controller board(DSP), 3.3V digital to the digital backplane, $+5 \mathrm{~V}$ to $-5 \mathrm{~V}$ for the analog board, the DSP program, DSP emulator, USB blaster cable, PC program and the PC. Oscilloscope is required for observing, monitoring and to enable us to control the signal sources to get the desired amplitude and frequency. Two silver needles are required for making EEG sources: object A and object B. Resistors are required to make the voltage divider circuit.

\subsection{Procedure Followed for Experimental Analysis}

Procedure for experimental analysis starts with a setup of the EEG+EIT IMM board along with the required power supplies for DSP board ( $5 \mathrm{v}$ digital power), digital back plane (3.3 v digital power), +5 to -5 analog power for the analog test board for a single IMM. Make all the connections correct and tight. Then a set up the two channels function generator has been done and the power supply is connected. Channel 1 is set for $100 \mu \mathrm{V}, 10$ $\mathrm{Hz}$ to get desired current across resistor $\mathrm{R}_{\mathrm{a}}$ and channel 2 has been set for $100 \mu \mathrm{V}, 25 \mathrm{~Hz}$ to get desired current across resistor $\mathrm{R}_{\mathrm{b}}$. Voltage signals are precisely monitored by the oscilloscope. EIT cylindrical phantom is connected on the table and is filled with the saline solution. Object A is constructed using two silver needles; needle electrode pair in one end, with the other end of the silver needles is exposed to the saline solution $(0.3 \mathrm{~S} / \mathrm{m})$. The two needles are separated by a distance of $10 \mathrm{~mm}$. Also object B is constructed in a same way as that of object A with the exposed ends resting inside the agar $(0.2 \mathrm{~S} / \mathrm{m})$ which is resting 
inside the saline $(0.3 \mathrm{~S} / \mathrm{m})$ solution. Whereas object $\mathrm{C}$ is constructed with agar only and no without any current injected to it. It is aimed at modeling the conductivity differences due to reasons other than electrical activity such as bleeding or something anomalous in the brain. Stand has been used to hold the object A and object B current injection sources vertically. Object $A$ is placed close to channel 9, object $C$ close to channel 16 and object $B$ in the middle of channel 4 and channel 5 with their locations correctly marked. A waveform of $1.125 \mathrm{KHz}$ is generated using the EEG+EIT IMM board and the current is injected in channel 16 using an electrode.

Data is acquired on each channel sequentially using the single EEG+EIT IMM board and processed using MATLAB to separate the modeled EEG signal and injected EIT signal frequency. FFT is plotted using MATLAB to observe the frequency spectrum of the acquired signal data. A low pass filter with cut off of 100 Hertz is used to get the modeled EEG data. A notch pass filter is used to filter the 60 hertz noise if there is any. Effective EEG signals are obtained from object A and object B on all the channels and their amplitude is plotted with respect to the channel number. Testing phantom of different testing objects is shown in Figure 6.

- Objecta:

\section{3 different testing objects}

- Brain activity as EEG source

- $10 \mathrm{~Hz}, 100 \mu \mathrm{V}$ closed to channel 9 of EIT system

- No conductivity change

- ObjectB:

- Brain activity as EEG source

- $25 \mathrm{~Hz}, 100 \mu \mathrm{V}$ close to channel 5 of EIT system

- Conductivity different $(0.2 \mathrm{Sm})$

- ObjectC:

- Nobrain activity

- Conductivity different frombleeding or other reasons $(0.2 \mathrm{Sm})$
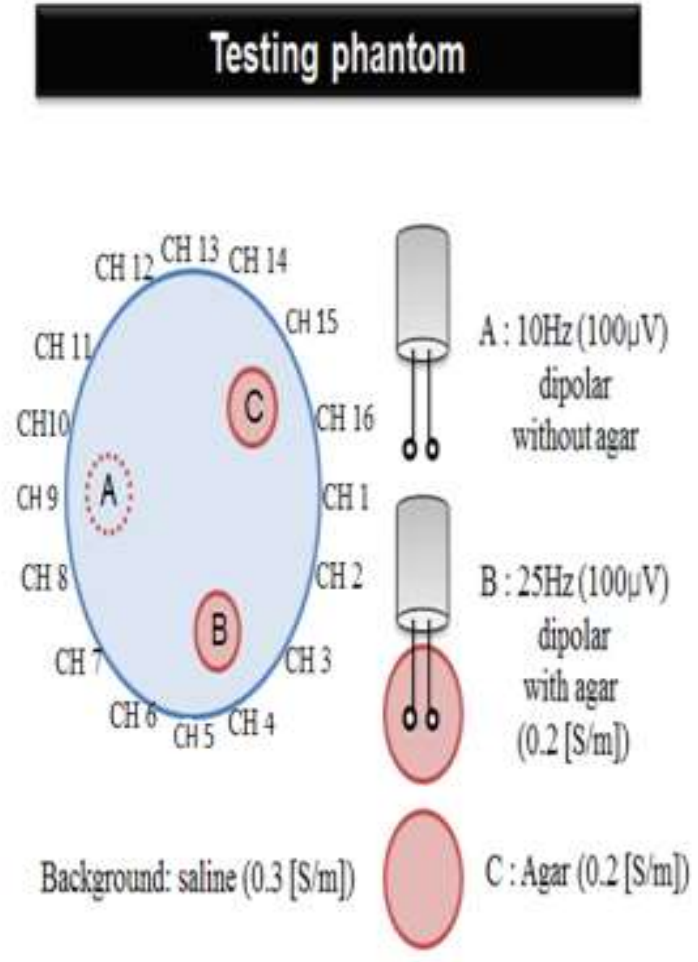

Figure 6. Test Phantom for Signal Measurement

\subsection{Pseudo EEG modeling and Measurement}

We modeled EEG signal as two sources of different frequencies and same amplitude of $100 \mu \mathrm{V}$. EEGsignal_1 is a source with $10 \mathrm{~Hz}$ frequency and is placed between channel 8 and 9 directly into the saline in the cylindrical phantom. EEG signal_2 has a frequency of $25 \mathrm{~Hz}$ and is placed between channel 3 and 4 and is actually placed in the agar medium, which in turn is placed into the saline solution. As the EEG Signal_1 is directly placed into the saline solution we expect to observe the larger measurement around channel 8 and 9 . Similarly as EEG Signal_2 is placed in the agar medium and then the agar is placed in the 
saline solution, the measurement around channel 3 and 4 is expected to be little smaller in magnitude as compared to in channel 8 and 9. The measured data is shown in Figure 7.

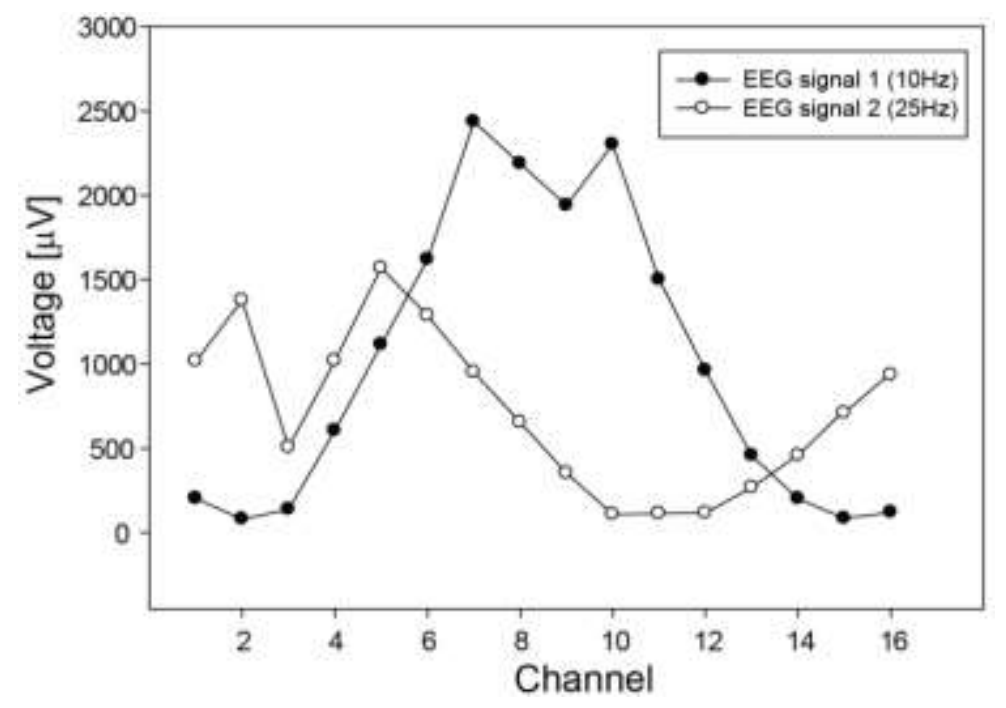

\section{Figure 7. Measured EEG Signal Voltage vs. the Channel no. of the Measurement Performed}

\section{Result and Discussion}

We have evaluated the function of new IMM in the saline phantom with different types of objects. We made the phantom model the EEG sources as already explained. Before applying EEG source signals $(10 \mathrm{~Hz}$ and $25 \mathrm{~Hz}$ on object $\mathrm{A}$ and $\mathrm{B})$, we measured EIT data for control image with two agar objects in the saline phantom. We recorded and compared the EIT images before and after applying EEG source signals. Images were found to be 99.99\% similar (calculated correlation coefficient). Deformation and localization error were in similar ranges i.e., below $1 \%$. From the measured data, we extracted $25 \mathrm{~Hz}$ and $10 \mathrm{~Hz}$ components. The magnitude of $10 \mathrm{~Hz}$ and $25 \mathrm{~Hz}$ from measured data is plotted in the graph with respect to measurement channel number. We observed that the $10 \mathrm{~Hz}$ signal is large around source region for EEG signal 1 i.e., around channel 8, 9. The $25 \mathrm{~Hz}$ signal is large around channel 3 and 4 which is the source region for EEG signal 2. Even though we applied same magnitude of $25 \mathrm{~Hz}$ signal to the Object B, we detected small $25 \mathrm{~Hz}$ signal refer to the $10 \mathrm{~Hz}$ signal because object $\mathrm{B}$ was surrounded by agar object $(0.2 \mathrm{~S} / \mathrm{m})$.

We upgraded the IMM with new ADC component, analogue filters, and amplifier circuits to acquire EEG and EIT signals together. We also implemented the timing control unit for generating injection signal and acquiring data with different sampling speed using internal PLL in the FPGA. Fast acquisition timing signal for EIT measurement, control signals for high resolution ADC for acquiring EEG signal, Phase sensitive demodulator and FFT logic for EIT acquisition was also performed using the FPGA. Post processing of the raw data for separating EEG signal from the combined signals with EIT was implemented in MATLAB. We have shown in Figure 8 that the EIT reconstructed image with EIT measurement only and with EIT+EEG simultaneous measurement comes out to be same. 


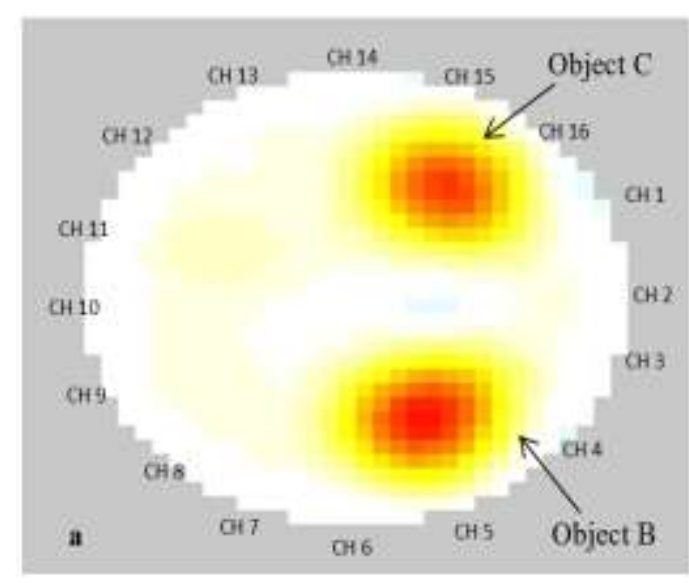

(a)

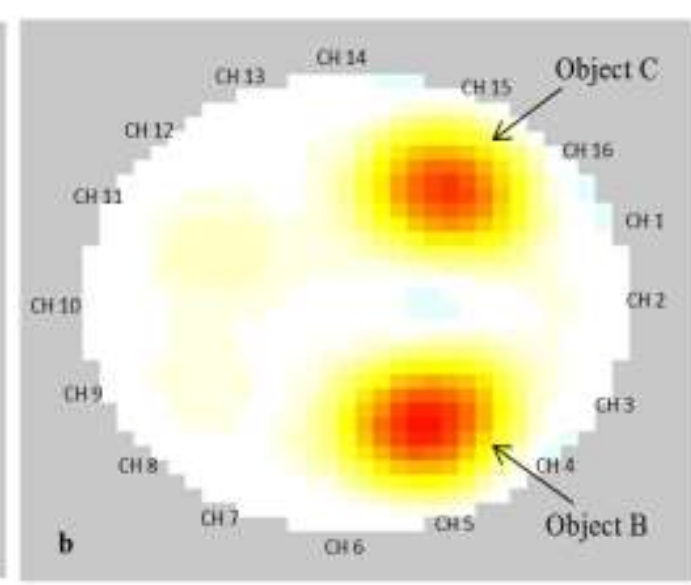

(b)

\section{Figure 8. EIT Reconstructed Images (a) Without EEG Signal Sources (b) with EEG Signal Sources}

\section{Conclusion and Future Scope}

Upgraded IMM is introduced with new ADC component, analogue filters, and amplifier circuits to acquire EEG and EIT signals together. Timing control unit is implemented in the FPGA for generating injection signal and fast acquisition timing signal for EIT measurement. Internal PLL is used for generating different sampling speeds. High resolution and wide sampling rate is $\mathrm{ADC}$ is used for acquiring low frequency EEG signal and comparatively higher frequency EIT signal simultaneously. Phase sensitive demodulator and FFT logic is also implemented in the FPGA for processing the acquired EIT signal. EEG data was separated from the EIT data using MATLAB software. Further EEG data can be processed while taking the EIT conductivity matrix obtained from EIT data processing as for solving the inverse problem of EEG source localization. Future work may concerns FPGA implementation of some post processing methods for separating EEG signal from the combined signals with EIT. Further communication speed between the experimental module and PC can be improved and timing control signal can be added for synchronous stimulation.

\section{Acknowledgments}

This project was supported by Prof. Eung Je Woo, Director IIRC, Kyung Hee University, Republic of Korea and Prof. Tong In Oh, Kyung Hee university, Republic of Korea. Authors would like to thank them for the facilities provided, useful discussions and guidance.

\section{References}

[1] G. Antoch and A. Bockisch, "Combined PET/MRI: a new dimension in whole-body oncology imaging?", European Journal of Nuclear Medicine and Molecular Imaging, vol. 36, no. 1, (2009), pp. 113-120.

[2] Y. Granot, A. Ivorra and B. Rubinsky, "Frequency Division Multiplexing for Electrical Impedance Tomography in Biomedical Applications", International Journal of Biomedical Imaging, (2007).

[3] S. I. Gonzalves, J. C. de Munck, J. P. A. Verbunt, F. Bijma, R. M. Heethaar and F. L. daSilva, "In Vivo Measurement of the Brain and Skull Resistivities Using an EIT-Based Method and Realistic Models for the Head", IEEE Transactions on Biomedical Engineering, vol. 50, no. 6, (2003), pp. 754-766.

[4] S. Ahn, S. C. Jun and E. J. Woo, "Numerical Simulation of Frequency-difference EIT using Multi-shell Concentric Spherical Head Model", 8th International Symposium on Noninvasive Functional Source Imaging of the Brain, (2011), pp. 1-4.

[5] G. Hahn, M. Beer, I. Frerichs, T. Dudykevych, T. Schroder and G. Hellige, "A simple method to check the dynamic performance of electrical impedance tomography systems", Physiol. Meas., vol. 21, (2000), pp. 53-60. 
[6] B. H. Brown, "Electrical impedance tomography (EIT): a review", Journal of Medical Engineering \& Technology, vol. 27, no. 3, (2003), pp. 97-108.

[7] A. J. Wilson, P. Milnes, A. R. Waterworth, R. H. Smallwood and B. H. Brown, "Mk 3.5: a modular, multifrequency successor to the Mk3a EIS/EIT system", Journal of Institute of Physics Publishing Physiological Measurement, (2001), pp. 49-54.

[8] M. Soleimani, "Electrical impedance tomography system: an open access circuit design", Biomedical Engineering Online, (2006).

[9] M. Soleimani, "Electrical impedance tomography imaging using a priori ultrasound data Medical Engineering On Line", (2006).

[10] T. I. Oh, E. J. Woo and D. Holder, "Multi-Frequency EIT System With Radially Symmetric Architecture: KHU Mark1", IoP Publishing, Physiol. Meas., vol. 28, (2007), pp. 183-196.

[11] T. I. Oh, H. Koo, K. H. Lee, S. M. Kim, J. Lee, S. W. Kim, J. K. Seo and E. J. Woo, "Validation of a multi-frequency electrical impedance tomography (mfEIT) system KHU Mark1: impedance spectroscopy and time-difference imaging”, IoP Publishing Physiological Measurement, Physiol. Meas., vol. 29, (2008), pp. 295-307.

[12] M. Goharian, M. Soleimani and A. Ravinthan, "A DSP Based Multi-Frequency 3D Electrical Impedance Tomography System", Annals of Biomedical Engineering, vol. 36, no. 9, (2008), pp. 1594-1603.

[13] J. Kuen, E. J. Woo and J. K. Seo, "Multi-frequency time-difference complex conductivity imaging of canine and human lungs using the KHU Mark1 EIT system”, IoP Publishing, Physiol. Meas., vol. 30, (2009), pp. 149-164.

[14] H. Sohal, W. Hun, A. L. McEwan, E. J. Woo and T. I. Oh, "Electrical impedance imaging system using FPGAs for flexibility and interoperability", Biomedical Engineering OnLine, (2014), pp. 2-14.

[15] X. Yue and C. McLeod, "FPGA design and implementation for EIT data acquisition", Iop Publishing Physiological Measurement, Physiol. Meas., vol. 29, (2008), pp. 1233-1246.

[16] T. Tang, S. Oh and R. J. Sadleir, "A Robust Current Pattern for the Detection of Intra ventricular Hemorrhage in Neonates Using Electrical Impedance Tomography”, Annals of Biomedical Engineering, vol. 38, no. 8, (2010), pp. 2733-2747.

[17] B. Esler, T. Lyons, S. Turovets and D. Tucker, "Instrumentation for low frequency EIT studies of the human head and its validation in phantom experiments", International Conference on Electrical Bio impedance, (2010), pp. 1-4.

[18] E. J. Woo, "Functional Brain Imaging using MREIT and EIT: Requirements and Feasibility", International Symposium on Bio electromagnetism, (2011), pp. 131-134.

[19] P. O. Gaggero, A. Adler, J. Brunner and P. Seitz, "Electrical impedance tomography system based on active electrodes", IoP Publishing, Physiol. Meas., vol. 33, (2012), pp. 831-847.

[20] W. Hun, H. Sohal, A. L. McEwan, E. J. Woo and T. I. Oh, "Multi-Frequency Electrical Impedance Tomography System With Automatic Self-Calibration for Long-Term Monitoring”, IEEE Transactions on Biomedical Circuits and Systems, vol. 8, no. 1, (2014), pp. 119-128.

[21] M. Michalikova and M. Prauzek, "A hybrid device for electrical impedance tomography and bioelectrical impedance spectroscopy measurement", IEEE Canadian Conference on Electrical and Computer Engineering, (2014), pp. 1-4.

[22] I. S. Sayed, "Modified PET / SPECT Cylindrical Phantom", World Congress on Medical Physics and Biomedical Engineering, (2006), pp. 1681-1683.

[23] B. Karki, H. Wi, A. McEwan, H. Kwon, T. In Oh, E. Je Woo and J. Keun Seo, "Evaluation of a multielectrode bioimpedance spectroscopy tensor probe to detect the anisotropic conductivity spectra of biological tissues", Meas. Sci. Technol., vol. 25, (2014), pp. 075702.

\section{Authors}

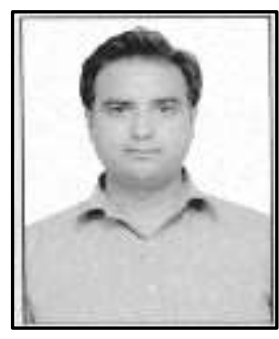

Dr. Harsh Sohal has received his is Ph. D. from Kyung Hee University, South Korea, while working as a research assistant in Impedance Imaging Research Center (IIRC) in the year 2014. Prior to that he did his M.Tech. in VLSI Design Automation \& Techniques from National Institute of Technology Hamirpur, India in 2008. He was awarded B. Tech. in Electronics \& Instrumentation Engineering by Punjab Technical University, Jalandhar, India in 2005. He has carried out research in interdisciplinary engineering disciplines in his career and has published several papers in SCI listed journals of repute. His research interests include medical device design, electronic instrumentation, hardware/software co-design, biomedical VLSI design, FPGA based instrumentation and algorithm implementation. 


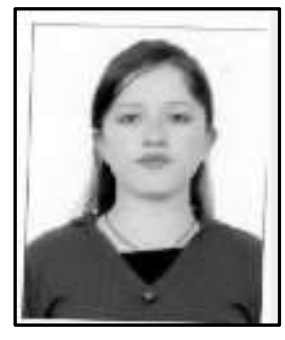

Anjali Sharma has received her B.Tech degree in Electronics and Communication engineering from Himachal Pradesh University, India in 2009 and ME degree in Electronics and Communication engineering from Panjab University, India in 2013. She is currently working towards the Ph.D. degree in Electronics and Communication engineering from Chitkara University Chandigarh, India. She has published more than 30 research papers in the field of Low Power VLSI design in International Journals and IEEE international conferences. Her current research interests include analysis and design of low power digital circuits, ultra-low power biomedical digital devices, low power considerations for nanotechnology and ultra-low power VLSI designs.

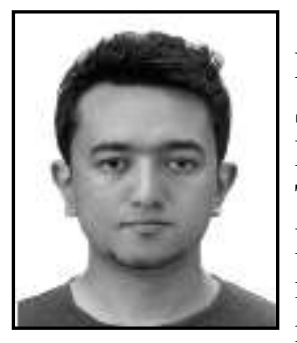

Bishal Karki was awarded Bachelor of Engineering in Biomedical Engineering from College of Biomedical Engineering and Applied Sciences, Nepal in the year 2009. He was awarded Masters in Biomedical Engineering from Kyung Hee University South Korea. There he worked as a graduate research assistant from 2011-2014in Impedance Imaging Research Center (IIRC). He has published articles in reputed journals for various applications of EIT. Attributed to his multidisciplinary research interests he is pursuing his doctorate degree in the field of Chemical and Process Engineering from the University Of Leeds, UK since 2016. His research interests include biomedical instrumentation, Electronics engineering etc.

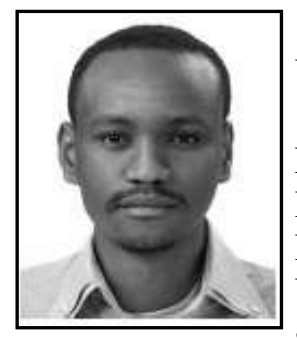

Dr. Youssoufa Mohamadou received his is Ph. D. from Kyung Hee University, South Korea, in the year 2014.

He did Bachelor of Science in Technical Education in Electrical and Electronic Engineering from Islamic University of Technology, Bangladesh in 2008. Prior to that he also completed his Higher Diploma in Electrical and Electronic Engineering from the Islamic University of Technology, Bangladesh in 2007. He has published several research papers in journals of repute. Currently he is working as Assistant Professor in Biomedical Engineering Department, Universite des Montagnes, Cameroon and has been instrumental in starting the process of establishing a research group in Electrical Impedance Tomography. He has carried out research in development of Electrical Impedance tomography based instrumentation systems design and evaluation. His research interests include electrical/electronic instrumentation and measurements, embedded systems development primarily for biomedical applications. 
International Journal of Control and Automation

Vol. 11, No. 9 (2018) 